\title{
Analysis of Management Strategy of MXBC
}

\author{
Jiayue Tang
}

\author{
Hefei No.8 High school \\ tjycissycom@163.com
}

\begin{abstract}
Milk tea is a kind of tea beverage which originated in Taiwan Province and has been popular since 1990s. It combines the fragrance of tea and the sweetness of milk, and has become one of the choices of people's daily drinks. Nowadays, with the increase of milk tea shops, the competitive of milk tea market begins to become greater. If a milk tea shop wants to have its own position in the whole milk tea market, it must have it own unique strategies. This article studies the most self-featured milk tea shop in the Chinese milk tea market. This article is mainly used to analyze several business strategies and reasons for the success of one of the milk tea shop-MXBC. Besides, by comparing with other milk tea shops, the author discovered the unique marketing strategy of MXBC. Finally, these strategies, such as expanding customer base, building their own stores, make MXBC become very successful. However, these strategies also bring some problems such as healthy problem. This article is used to those who want to learn more about the milk tea industry, and get more knowledge about MXBC.
\end{abstract}

Keywords: $M X B C$, Business strategy, Competitive advantage, Enterprise

\section{INTRODUCTION}

In recent years, the rise of milk tea industry has increased the competitive pressure of the whole market. According to the data of the First Financial Business Data Center, the growth rate of tea in China was the highest from 2018 to 2019, which increased by $200 \%$ compared with the same period of last year. Tea drinks have gradually become the favorite drinks of consumers. Now there are many different brands of milk tea in the market, and new brands will enter this market in the future. Faced with such great competitive pressure, MXBC gets its own market. MXBC is one of Chinese milk tea shops, and it is very successful to have its own competitive advantage compared to other milk tea shops. In 2021, there were more than 10,000 offline stores in the country. This article wants to explore the reasons why MXBC has a place and the recent changes. By comparing with other types of tea shops, it reflects the advantages and some drawbacks of MXBC itself. The article helps people to better understand the operation mode of MXBC. MXBC has created a brand-new chain form, integrating high quality and low price with health and freshness to provide consumers with special products[1]. This article will explain why MXBC has been loved by customers in a short period of time and the reasons for its success in the milk tea market. At the same time, the management model of
MXBC also has brought some bad influence, which the author will also discuss.

\section{OVERVIEW OF MXBC BUSINESS STRATEGIES}

\subsection{Management strategy}

MXBC adopts the management strategy of small profits but quick turnover. First of all, all the products in MXBC are considered as low prices in the market. Obviously, the commodity price of MXBC is generally lower than that of other milk tea shops. For example, in Hefei, Anhui Province, China, the price of goods at any MXBC is almost no more than 10 yuan. With bubble tea as a comparison, a large cup of milk milk tea is only 9 yuan in MXBC, but in other milk tea shops in Hefei, Coco needs 11 yuan, A little-tea needs14 yuan, and R and $\mathrm{b}$ club needs 11 yuan. By comparison, it can be seen that MXBC has a significant price advantage. Besides, MXBC is aimed at students and some workers who have some low wage, so the price in MXBC is popular for these people. Then, the author will discuss the reasons for the low price of MXBC.

The reason is that the low cost of MXBC. MXBC saves its costs in several ways. Firstly, MXBC has its own research team and warehousing logistics. The 
existence of these two things helps the city save a lot of money. To be more specific, MXBC has a unique product research and development team, independent research and development of good products. Selection of global quality raw materials can achieve the core raw materials of self-production, and has a number of patented technologies. It can be easily found that the independent research and development team enables MXBC to spend less money to launch and develop new products compared with other milk tea shops. In addition, they do not need to spend a high price to rent other processing plants to produce milk tea raw materials. Then, MXBC establishes its own warehousing and logistics centers throughout the country, with Zhengzhou as the center, covering the whole country from point to point, and implies the logistics free shipping policy. This can reduce the cost of finding a logistics company, and improve the speed of shipping in all places, effectively improving. The last way to reduce costs is to rent a small facade shop. It is easy to find that you can hardly see the shadow of the MXBC in big shopping malls and some high-spending shopping malls. However, MXBC often chooses some small shops along the street. The reason of this phenomenon is that small shops have lower electricity bill and rent so it is a good way to reduce the cost.

\subsection{Expand customer base through multiple channels}

MXBC will increase its sales in many ways. MXBC often held some activities from time to time to attract customers, and often introduce new products to meet customers who like different tastes. For example, recently, due to the arrival of hot summer, MXBC launched a new peach rocking music product. Sweetie milkshake and smoothies make many customers feel fresh and loved. Besides, the buy one gets one free activity just three days after the opening of MXBC. Using the admission ticket for college entrance examination can offer discounts and other activities, which make more customers come to the ice city for consumption. Then, there's also the theme song of MXBC, which was very popular some time ago. The melody of this song is monotonous and the lyrics are interesting, so it's easy for people to remember. Interesting song is also an effective way to make more people know about the MXBC. Apart from this, MXBC has its own unique internet marketing: take-out sales activities are carried out by using online discount and group buying platforms such as Meituan. Selling through the take-out platform allows customers who are far away from the store to enjoy delicious milk tea. At the same time, selling through the take-out platform also brings great convenience to some inconvenient and lazy customers who go to the store to order food.

\subsection{Increase stores}

MXBC increases its stores and improve its popularity and competitiveness. In 2021, MXBC has 12,000 branches, and MXBC covers 31 provinces across the country. More and more people have the opportunity to taste MXBC in this way. Besides, Honey MXBC has its own special product--ice cream. Ice cream in Honey MXBC is always a hot product. Honey MXBC also has some details on ice cream. For example, in March 2007, Honey MXBC gave up the traditional plastic wrapping paper that polluted the environment and switched to environmentally friendly recycled paper. Some of the milk used in ice cream in Honey MXBC comes from New Zealand. The high-quality milk source breaks people's impression that the low price of MXBC leads to poor quality. MXBC cooperates with Fonterra Dairy Group, the largest brand in New Zealand, to ensure that the milk consumed is fresh. In terms of ensuring food consumption, MXBC has also introduced ice cream rocking music, which allows customers to fully blend the materials in the cups by shaking. This special game is also popular with customers.

\section{NEGATIVE EFFECTS OF MARKETING STRATEGY}

However, these business strategies will also bring some negative impacts to the Ice City of Honey Snow.

\subsection{The range of customers is limited}

First of all, in order to reduce their own operating costs, most of the shops in MXBC often choose some small shops on the roadside. As a result, there is no way for MXBC to open high-consumption shopping malls, because renting shops in those places is too expensive for them, even if there is a large flow of people. In addition, the low consumption of MXBC makes some administrators of high-consumption shopping malls prohibit MXBC from renting their stores. MXBC usually chooses to open its shops next to some chain stores, small shops next to scenic spots and places with a large number of bus stops, but they will not choose shopping malls with high consumption. Thus, it is difficult for MXBC to win the favor of some more upscale people. Renting a small shop will limit its own consumer groups to workers and students with lower wages.

\subsection{Branch traffic is getting smaller}

The second problem is that with the rapid expansion of MXBC, the distance between each branch and each branch is getting closer and closer. The periphery of the school and the business district are destined to be limited. Now, the expansion of the branch in MXBC has diverted the flow of people, resulting in fewer and fewer 
customers in each branch, and it is increasingly difficult for the branch administrators to make money. According to the description of the staff of MXBC, there are now 13 branches of MXBC in Tongzhou, Beijing. It is not uncommon for two or three ice cities with honey snow to appear on one street at the same time. Therefore, with the rapid expansion, many branches have gone bankrupt. In addition, the MXBC, which is established in the same area, has a regional manager. With more and more branches, regional managers have more shops to manage and more problems to worry about. Regional managers need to spend more time to supervise the hygiene of each branch and answer some other business questions of branch owners. Taking care of the shops for a long time will cause regional managers to be overworked, and finally give up the supervision of some branches, which is one of the reasons for the health problems in the MXBC some time ago. Due to the improper management of regional managers, some employees' early training is unqualified, but in order to improve the efficiency of training, regional managers still choose to let these employees take up their posts.

\subsection{Health problems}

Another cause of health problems in MXBC is small profits. On May 15th of this year, several branches in MXBC were inspected by inspectors to tamper with food safety problems in Zhengzhou City, using overnight tea, milk and broken fruits [2]. A shop owner of the MXBC branch said that in one post that if shop owner can earn enough money, they may not choose to use these unhealthy food. Nowadays, the rent, labor cost and take-out charge is very high and unit price of products is very low so shop owners can't get enough profit. Then, most of them choose so save some cost through using some low quality of food or using outdated food. Although the official data given by MXBC is the gross profit can be about $50 \%$ and net profit can achieve $20 \%$, the shop owners say that they can't get this standard because of low unit of products. In this branch manager's view, MXBC Head Office only wants to expand fiercely, and does not care whether these branch managers can make money. Low profit leads to fewer and fewer people willing to manage the branch of MXBC, which leads to food safety becoming a hidden danger.

\section{DISCUSSION}

With regard to the problem of too many branches, MXBC can stop expanding and strictly manage the existing stores. Strictly management can let more customers trust the brand of MXBC, and become more comfortable with health problems. MXBC now strictly requires employees to throw away fruits that have stayed overnight, not to use milk that has been left for a long time, and impose some fines on them if they find violations. Only punishment will make employees remember these rules. On the issue of expanding the scope of customers, the only way to solve the problem of peak traffic is to find new traffic. Now there are two ways to go, one is to attack the high-end market that has not been considered yet. As mentioned above, the MXBC rarely opens its own shops in high-consumption and high-end shopping malls, which can be considered for attack in the future. Second, the products are more diversified, and more product categories are expanded to meet the needs of more customers. Similarly, in order to attract more customers, activities are also very important. People can hold some preferential activities on some festivals such as Valentine's Day and Christmas. Online activities are also possible for people to participate more conveniently.

\section{CONCLUSION}

Overall, the small profits but quick turnover management mode and the expansion of its own shops have made MXBC have its own position in the milk tea market, and are deeply loved by customers. However, these modes of operation will also have many negative effects. For example, the operating pressure of branch stores becomes greater, and the requirements for regional administrators become higher, which eventually leads to some health problems. The lack of enough traffic is the main problem that MXBC is facing now. This article has no way to include the problems of the MXBC in all regions, and it has no way to predict the future development of the MXBC too much. Future research will mainly focus on some adjustments and improvements made by $\mathrm{MXBC}$ in the face of these problems. Besides, here is no way to obtain the annual profit report of MXBC, and there is no way to analyze the relative data. In addition, a lot of information comes from the words of MXBC employees, and it is inevitable that there are no subjective factors. In the future, we may do more research in statistics and economics, and analyze more different types of stores.

\section{ACKNOWLEDGMENTS}

Firstly, I would like to show my deepest gratitude to my teachers who have provided me with valuable guidance in every stage of the writing of this thesis. Further, I would like to thank some employees who work in MXBC for the information that they provided, and the information help me complete my thesis. In the end, I would like to thank my parents for their encouragement and support. Without all their enlightening instruction, Enthusiastic help and impressive kindness, I could not have completed my thesis.

\section{REFERENCES}

[1] Official website of MXBC, https://www.mxbc.com/ 
[2] Tencent News, May21,2021, MXBC: The Use of Expired Ingredients, Unqualified Disinfection and Ineffective Management System, https://new.qq.com/rain/a/20210517A0A8KN00

[3] Chen,J, MXBC and Heytea, Which One Would be The Next Trending drink? Modern Advertising,8th issue,pp.24-25,2021:http://qikan.cqvip.com/Qikan/ Article/Detail?id=7104606859\&from=Qikan_Searc h_Index )

[4] Lang W, Shi D, MXBC is Caught in The Food Safety Storm, Which is Caused by The Rapid Expansion, Business Management Review, 6th issue ,pp.25-28,2021:http://qikan.cqvip.com/Qikan/Articl e/Detail?id=7104883659\&from=Qikan_Search_Ind ex

[5] Liu Yayu. Marketing Strategy Analysis of MXBC in Zhengzhou City, China Management Informationization, 15th issue, pp.70-71, 2015:http://qikan.cqvip.com/Qikan/Article/Detail?i d=669949571\&from=Qikan_Search_Index )

[6] Wang Y, Exploration of Innovative Marketing Mode of Traditional Catering Industry in Internet Era Based on the Successful Marketing Case of Little Milk Tea, Foreign Economic Relations and Trade , 9th issue pp.106-108,2019:http://qikan.cqvip.com/Qikan/Art icle/Detail?id=7100373356\&from=Qikan_Search_I ndex

[7] Ding Xiaobing, Kanglai's Cross-border Milk Tea Business, Knowledge Economy, 9th issue, pp.48-51,2021:http://qikan.cqvip.com/Qikan/Articl e/Detail?id=7105541717\&from=Qikan_Search_Ind ex

[8] Development $\mathrm{Xu}$ Jiamin, Wang Xiaoyu, Liang Fangchu. Research on Consumption Status and Strategy of Little Milk Tea Based on SWOT Model, Modern Marketing: Business Edition, 4thissue,pp.36-37,2021:http://qikan.cqvip.com/Qik an/Article/Detail?id=7105331340\&from=Qikan_Se arch_Index 